\title{
Structure and Spectral Characteristics of Glucose
}

\author{
Ran Song ${ }^{1}$, Dong Chen ${ }^{1, *}$ Chengxiang Suo ${ }^{1}$, Zhiheng Guo ${ }^{1}$ \\ ${ }^{1}$ Physics and Electronics Department, Henan University, Kaifeng 475004, China
}

\begin{abstract}
Compared with the traditional methods, the fully random conformational search based on Monte Carlo is more random and comprehensive. In this paper, the structural characteristics and spectral vibration of glucose molecules are studied by this method, and the results are compared with the existing results, which are highly consistent in all aspects, confirming the scientificity and accuracy of this method. At last, the properties, functions and spectral vibrations of glucose are studied by combining electrostatic analysis and spectral analysis. This method provides a new way to study the related properties of other biomolecules.
\end{abstract}

\section{Introduction}

In 1747, S. Marggraf first isolated glucose in Berlin. As a direct energy substance for life activities, glucose does not need to be hydrolyzed to participate in the metabolism of the body. It can directly provide a continuous supply of energy for life activities to meet the normal survival needs of the body [1, 2]. In addition, glucose also plays an important role in clinical treatment. It is not only the medium of intravenous nutrient input, constantly adjusting the osmotic pressure stability of cell fluid, but also can treat hypoglycemia, edema, hyperkalemia and other diseases. Therefore, our normal life, learning, work and medical treatment are inseparable from this small glucose molecule [3-6]. We all know that there are many kinds of monosaccharides with the same chemical formula as glucose molecules, but their chemical properties are quite different from each other[7]. Therefore, in order to understand the characteristics of glucose, it is necessary to study the spatial structure of glucose in depth. This paper will provide a new method to study the spatial structure of biomolecules, which is called fully random conformational search based on Monte Carlo [8, 9]. This method combined with molecular dynamics can search out all possible spatial structures of glucose according to the basic model of glucose. In this process, we have tried many times to determine the most reasonable experimental parameters in the search, so as to ensure the rationality and accuracy of the final results.

The research content mainly focuses on the molecular structure, intramolecular hydrogen bond and spectral vibration characteristics. As a strong non-bonded interaction in the biomolecular system, hydrogen bond has a great impact on the structural characteristics and chemical properties of biomolecules [10-15]. So it is necessary to study the hydrogen bond interaction.

\section{Computational methods}

In this paper, the research on the spatial structure of glucose starts from fully random conformational search of monosaccharide structure. Through Monte Carlo method and MMFFS force field [16-17] in MacroModel 11.2, we conducted 1000 times of configuration search to generate the initial configuration of glucose molecule. Because the chair framework supports the whole structure, we fixed the chair structure of glucose ring during the configuration search, set the torsional rotation value of hydroxyl group and hydroxymethyl group on the sugar ring to $0^{\circ}-180^{\circ}$, and change each bond that allows rotation in 100 steps. The specific parameter settings are shown in Fig. 1.

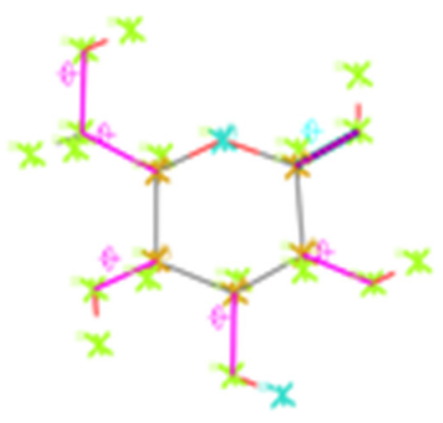

Fig. 1. Full random conformational search parameter settings of glucose.

After the above parameters are set, the energy window is set to $50 \mathrm{~kJ} / \mathrm{mol}$, which can filter out some high-energy configuration, reduce the difficulty of calculation work, and improve the work efficiency. Among the 627 structures generated in this search process, 68 structures are finally determined to carry out the quantum chemical calculation. After the preliminary optimization of these structures, some configurations will be screened again. Then, the representative configuration of glucose will be obtained by single-point energy calculation, zero-point 
energy correction and frequency analysis. The process of the operation is shown in Fig. 2.

\section{Stagel: Construct initial structure \\ Operation 1: Fully random conformational search \\ Stage2: Search all possible structures

$$
\begin{aligned}
& \text { Operation 2: Choose the right } \\
& \text { configuration }
\end{aligned}
$$ \\ Stage3: Perform quantum chemical calculations$$
\text { Operation 3: High-order energy }
$$ \\ calculation and analysis \\ Stage4: Determine representative configuration}

Fig. 2. Operation process description.

In the above related quantum chemistry calculation, all the calculation tasks are completed by gaussin-09 software. The b3lyp/6-311++g (d, p) method and base group are selected to complete the calculation task [18-20]. Finally, the results are analyzed, which is confirmed that the spatial structure of glucose is highly consistent with the existing data.

\section{Results and discussion}

\subsection{Structure analysis of glucose}

Through analysis, we finally select 10 configurations as representative configurations with relative energy in the range of $20 \mathrm{~kJ} / \mathrm{mol}$. The relative energy values and spatial structure is shown in Fig. 3. For the convenience of subsequent description, we code the carbon atom and oxygen atom in glucose uniformly, and name the oxygen atom on the sugar ring as the fifth oxygen atom. Based on this oxygen atom, the clockwise coding carbon atom is successively named as 1 5 carbon atom, and the carbon atom in hydroxymethyl is named as the sixth carbon atom, and it is also stipulated that the number of the oxygen atom directly connected to the carbon atom is the same as that of the carbon atom.

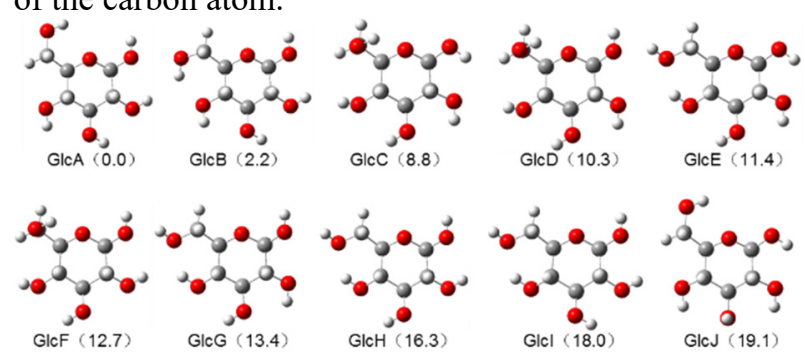

Fig. 3. Low-energy conformers of glucose.

From Fig. 3, we can see that the relative energy difference between GlcA and GlcB is about $2.21 \mathrm{~kJ} / \mathrm{mol}$, while the relative energy difference between GlcB and $\mathrm{GlcC}$ is about $6.61 \mathrm{~kJ} / \mathrm{mol}$, which indicates that $\mathrm{GlcC}$ is not as stable as GlcA and GlcB. We can find that both GlcA and GlcB have stable cooperative hydrogen bonding, so their structures are more stable and their energy is relatively low. Although there is also cooperative hydrogen bonding in GlcC, the direction of hydroxymethyl is perpendicular to the whole sugar ring plane, which results in weak hydrogen bonding between hydroxymethyl and $\mathrm{O}_{5}$. Therefore, the stability of $\mathrm{GlcC}$ is poor compared with GlcA and GlcB, so the relative energy increases sharply. When carefully comparing the structural differences between GlcA and $\mathrm{GlcB}$, the cooperative hydrogen bonding of the former is between $\mathrm{OH}_{1}$ and $\mathrm{OH}_{4}$, while the latter is between $\mathrm{OH}_{1}$ and $\mathrm{OH}_{6}$. [21]. What is more, the hydrogen bond of $\mathrm{OH}_{6} \rightarrow \mathrm{O}_{5}$ is formed in GlcA, while the hydrogen bond of $\mathrm{OH}_{6} \rightarrow \mathrm{OH}_{4}$ is formed in GlcB. The energy difference between them is $2.21 \mathrm{~kJ} / \mathrm{mol}$, indicating that the hydrogen bond of $\mathrm{OH}_{6} \rightarrow \mathrm{O}_{5}$ is more stable than $\mathrm{OH}_{6} \rightarrow \mathrm{OH}_{4}$. Observing the characteristics of other structures in Fig. 3, we can see that there is no overall cooperative hydrogen bonding except for the GlcE, so the relative energy of structural is generally increased. Although there is a cooperative hydrogen bond in GlcE, the internal hydroxymethyl group, as one of the most important functional groups of glucose molecules, will directly lead to different types of hydrogen bonds formed in the molecules due to different spatial orientations. As an important non-covalent interaction in biomolecules, hydrogen bonds directly affect the structure and stability of biomolecules [22-27], so the orientation of hydroxymethyl makes the whole molecular structure of GlcE extremely unstable, so the energy is also increased. The specific values of relative energy and spatial structure characteristics of other configurations can be obtained from Fig. 3.

When we compare the results of the fully random conformational search with existing data, we find that the results are highly consistent, which also verifies the rationality and scientificity of the method. It provides a research plan and a theoretical basis for our follow-up study.

\subsection{Raman analysis of glucose}

As shown in Fig. 4, the Raman vibration spectrum of GlcA and GlcB in the range of $2850 \mathrm{~cm}^{-1} \sim 3900 \mathrm{~cm}^{-1}$ are selected. Comparing the differences from the figure, we find that the number of vibration peaks is seven before $3200 \mathrm{~cm}^{-1}$, and the frequency positions of each peak are almost the same. When we take this feature into account the structure and composition of glucose, we will find that those peaks come from the vibration of hydrogen atoms directly connected with carbon atoms. In detail, these vibration peaks are generated by the vibration of five hydrogen atoms on the sugar ring that are directly connected to the carbon atom and two hydrogen atoms in the hydroxymethyl that are directly connected to the carbon atom. Because we find that no matter how the structure of the two kinds of glucose changes, the chair skeleton contained in them is the same, so the atomic vibration on the structure is similar. Therefore, it is easy to understand that there is a high consistency of the Raman vibration peak in this range. The main difference between GlcA and 
$\mathrm{GlcB}$ is the position of the vibration peak in the range of $3700 \mathrm{~cm}^{-1} \sim 3900 \mathrm{~cm}^{-1}$. The vibration peak in this range comes from the vibration of the hydroxyl group in glucose. The two representative structures of glucose have five hydroxyl groups respectively, corresponding to the five vibration peaks in their respective ranges.

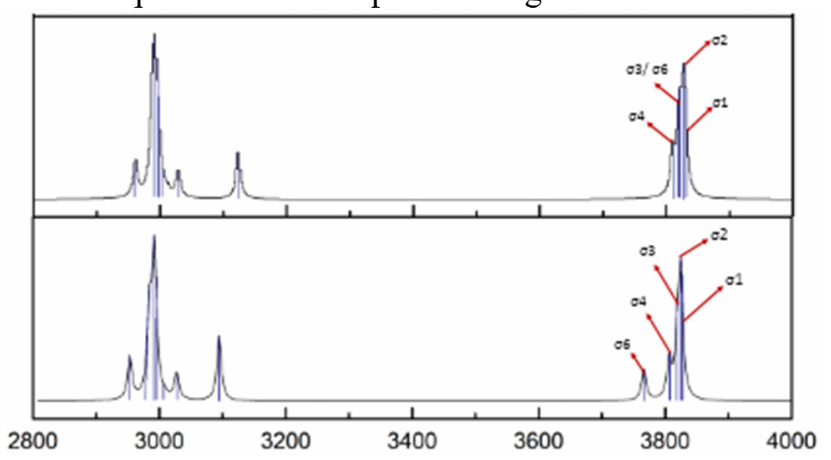

Fig. 4. Raman spectra of GlcA and GlcB

The on $(\mathrm{n}=1,2,3,4,6)$ in the figure above respectively corresponds to the vibration positions of the five hydroxyl groups in the glucose molecule. When we carefully observe the vibration positions of the five peaks, we can see that the vibration peak of $\sigma 1, \sigma 2, \sigma 3$ and $\sigma 4$ are basically the same, and the main difference is the vibration of $\sigma 6$, which corresponds to the vibration of $\mathrm{OH}_{6}$ on the glucose. The $\mathrm{OH}_{6}$ vibration peak in GlcA is located at $3819.60 \mathrm{~cm}^{-1}$, the peak value of $\mathrm{OH}_{6}$ in $\mathrm{GlcB}$ is 3766.16 $\mathrm{cm}^{-1}$. Compared with GlcA, the peak value of $\mathrm{OH}_{6}$ in $\mathrm{GlcB}$ has a red shift of $53.44 \mathrm{~cm}^{-1}$. The reason for this difference is that the direction of hydroxymethyl directly affects the vibration of hydroxyl group. These phenomena are also consistent with our previous discussion.

\section{Conclusion}

In this paper, we mainly study the fully random conformational search based on Monte Carlo to study the molecular space structure of glucose. Through a series of screening and quantum chemical calculation, we finally determine the two most representative glucose molecular structures, GlcA and GlcB. The similarities and differences between the two representative configurations are compared from configuration and spectral vibration analysis. It is confirmed that the fully random conformational search based on Monte Carlo can be used to study the spatial structure and property function of molecules, which also lays a theoretical foundation for further research.

\section{Acknowledgments}

The research was supported by the Chinese Postdoctoral Science Foundation under Contract No. 2017M612386,
Natural Science Foundation of Henan Province under Contract No.162300410011.

\section{References}

1. B. Ross, J. Espinal, P. Silva, Kidney Int, 29, 54 (1986)

2. F. Assimacopoulos-Jeannet, I. Cusin, R.M. GrecoPerotto, Biochimie, 73, 67 (1991)

3. Y. Saito, I. Takahashi, K. I. wane, 28, 569 (2013)

4. C. Voss, C. Lorenz, L. Ratzmann, Acta Biol Med Ger, 33, 515 (1974)

5. J. Ward, Clin Physiol, 5, 85 (1985)

6. S. Gapstur, P. Gann, JAMA, 283, 2552 (2000)

7. P.G. Seybold, U.A. Bagal, JCE, 64, 575 (1987)

8. E.J. Cocinero, E.C. Stanca-Kaposta, M. Dethlefsen, Chem, 15, 13427 (2009)

9. I. Hünig, A.J. Painter, R.A. Jockusch, PCCP, 7, 2474 (2005)

10. L. Brammer, E. Bruton, P. Sherwood, Cryst Growth Des, 1, 277 (2001)

11. M. Cowan, B. Bruner, Nature, 434, 199 (2005)

12. G. Gilli, P. Gilli, J Mol Struct, 552, 1 (2000)

13. R. Vargas, J. Garza, D. Dixon, ACS, 122, 4750 (2000)

14. S. Katsyuba, M. Vener, E. Zvereva, JPC, 117, 9094(2013)

15. L. Ramaniah, K. Chinnathambi, R. Kshirsagar, Mol Phys, 111, 5 (2013)

16. E.J. Cocinero, D.P. Gamblin, B.G. Davis, ACS, 131,11117 (2009)

17. T. Ping-hui, Y. Yu-heng, L. Yin-li, J Mol Struct, 894, 9 (2009)

18. N. Mayorkas, S. Rudi, B. Davis, Chem Sci, 2,3 (2011)

19. J. Tirado-Rives, W. Jorgensen, Mol Phys, 4, 297 (2008)

20. M. Wodrich, C. Corminboeuf, P. Schleyer, Org Lett, 8, 3631 (2006)

21. G. Jianming, C. Dong, S. Ran, Comput Theor Chem, 1, 24 (2018)

22. S. Simon, M. Duran, J.J. Dannenberg, J Chem Phys, 105, 11024 (1996)

23. M.M. Feldstein, P.E. Kireeva, T.I. Kiseleva, Poly Sci A, 51, 799 (2009)

24. C. Clarke, R.J. Woods, ACS, 123, 12238 (2001)

25. T. Steiner, Angew Chem, 41, 49 (2002)

26. M.S. Taylor, E.N. Jacobsen, Angew Chem Int Edit, 45, 1520 (2006)

27. C.J. Feck, J.D. Eaves, Science, 301, 1698 (2003) 